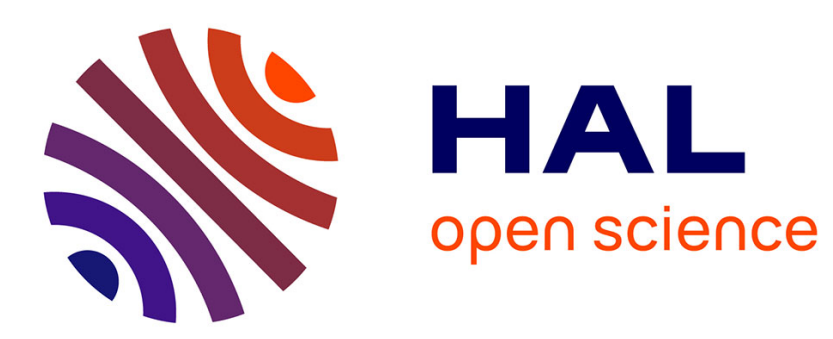

\title{
Efficient allocation algorithms for multicarrier spread-spectrum schemes in UWB applications
}

\author{
Antoine Stephan, Jean-Yves Baudais, Jean-François Hélard
}

\section{To cite this version:}

Antoine Stephan, Jean-Yves Baudais, Jean-François Hélard. Efficient allocation algorithms for multicarrier spread-spectrum schemes in UWB applications. IEEE International Conference on Ultra-Wideband (ICUWB'07), Sep 2007, Singapour, Singapore. pp.551-555, 10.1109/ICUWB.2007.4381006 . hal-00194983

\section{HAL Id: hal-00194983 \\ https://hal.science/hal-00194983}

Submitted on 7 Dec 2007

HAL is a multi-disciplinary open access archive for the deposit and dissemination of scientific research documents, whether they are published or not. The documents may come from teaching and research institutions in France or abroad, or from public or private research centers.
L'archive ouverte pluridisciplinaire HAL, est destinée au dépôt et à la diffusion de documents scientifiques de niveau recherche, publiés ou non, émanant des établissements d'enseignement et de recherche français ou étrangers, des laboratoires publics ou privés. 


\title{
Efficient Allocation Algorithms for Multicarrier Spread-Spectrum Schemes in UWB Applications
}

\author{
Antoine Stephan, Jean-Yves Baudais and Jean-François Hélard \\ Institute of Electronics and Telecommunications of Rennes (IETR) \\ INSA, 20 Avenue des Buttes de Coesmes, 35043 Rennes, France \\ antoine.stephan@ens.insa-rennes.fr, \{jean-yves.baudais ; jean-francois.helard\}@insa-rennes.fr
}

\begin{abstract}
In this paper, we propose a multicarrier spread spectrum (MC-SS) waveform for high data rate UWB applications, taking into account the American and the European UWB contexts. This new waveform for UWB is presented as an evolution of the well known Multiband OFDM Alliance (MBOA) solution and does not increase the system complexity significantly. First, we optimize the number of spreading codes to maximize the system range for a fixed QPSK constellation. Secondly, we use variable constellation orders and we propose a low-complexity resource allocation algorithm that maximizes the system throughput. We show that our adaptive MC-SS system transmits information at much higher attenuation levels and with higher throughput compared to the MBOA solution, and can be advantageously exploited for UWB applications.
\end{abstract}

Index Terms - Multiband OFDM, multicarrier spreadspectrum (MC-SS), resource allocation, UWB.

\section{INTRODUCTION}

Ultra-wideband (UWB) technology has attracted considerable interest in short range, high data rate wireless communications since 2002 when the Federal Communications Commission (FCC) agreed on the allocation of a 3.1-10.6 GHz spectrum for unlicensed use of UWB devices [1]. The FCC regulations are very stringent and require very low transmitted power since the UWB spectrum overlays other existing spectrum allocations.

One of the multiple-access techniques considered by the IEEE 802.15.3a standardization group is a multiband orthogonal frequency division multiplexing (MB-OFDM) supported by the Multiband OFDM Alliance (MBOA) [2]. Some studies have proposed to use a multicarrier codedivision multiple-access (MC-CDMA) waveform by adding a CDMA scheme to the OFDM solution with a spreading in the frequency domain, in order to improve the system performance [3]. In this paper, we propose a multicarrier spread-spectrum (MC-SS) scheme, known as linear precoding OFDM (LP-OFDM), which respects the OFDM parameters of the MBOA solution in order not to increase the system complexity significantly. This MC-SS technique improves the signal robustness against frequency selectivity and narrowband interferences.

An analytical study focusing on the optimization of the spreading function is presented. More precisely, the intrinsic characteristics and capabilities of the spreading function are highlighted and allocation algorithms are developed to improve the system performance. Two cases are considered for the optimization study. First, we consider a fixed quadrature phase-shift keying (QPSK) constellation as in the MBOA solution, and we find the optimal spreading code length and number of codes that maximize the noise margin, and consequently the system range. Secondly, in order to further improve the system throughput, we consider variable constellation orders as QPSK, 8-QAM (quadrature amplitude modulation) and 16-QAM. The UWB channel response varies slowly in time and can be considered quasi-static. Hence, we assume that the channel state information (CSI) is available at the transmitter. We propose a low-complexity resource allocation algorithm that distributes energy, bits and codes efficiently across the spectrum in order to achieve higher throughput, under a power spectral density (PSD) constraint. Simulations are performed by considering the American and the European UWB context. The optimization study does not take into account the channel coding scheme, but the performance comparison of the final systems with channel coding shows that the adaptive MC-SS can be advantageously exploited for UWB applications

This paper is organized as follows. Section II presents the proposed MC-SS scheme. Section III details the MC-SS optimization study and describes the proposed resource allocation algorithms, first in the case of a fixed QPSK constellation and secondly in the case of variable constellation orders. Simulation results showing the interest of the proposed adaptive scheme in UWB applications are given in Section IV, followed by the conclusion in Section V.

\section{SYSTEM MODEL}

The MBOA solution is based on the combination of an OFDM modulation with a multiband approach that divides the $7.5 \mathrm{GHz}$ UWB spectrum into 14 sub-bands of $528 \mathrm{MHz}$ each. The OFDM scheme consists of 128 subcarriers, out of which 100 are assigned to data tones. The constellation used is a QPSK, which leads to the transmission of 200 data bits per OFDM symbol. Initially, most of the studies have been performed on the first 3 sub-bands $(3.1-4.8 \mathrm{GHz})$. The FCC imposed a PSD limit of $-41.3 \mathrm{dBm} / \mathrm{MHz}$ on all the 14 sub-bands. With the European Electronic Communications Committee (ECC) regulations of March 2006, much lower PSD limits were imposed on the UWB spectrum, except on 


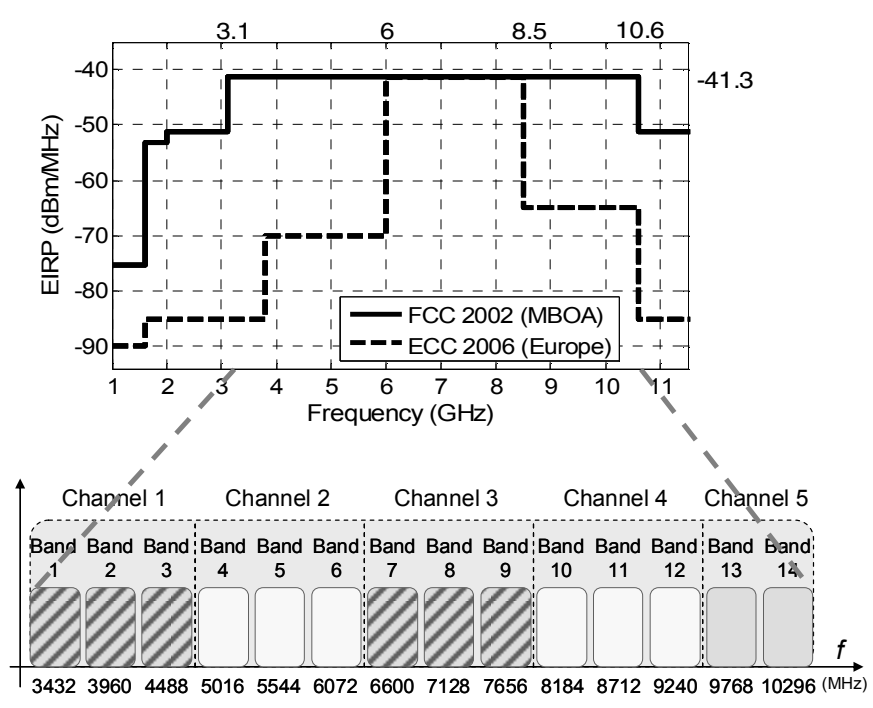

Fig. 1. PSD mask (FCC and ECC) and sub-bands distribution of the MBOA solution.

the $6-8.5 \mathrm{GHz}$ range where a similar limit value of -41.3 $\mathrm{dBm} / \mathrm{MHz}$ was considered [4]. Hence, when we consider the European context, we perform our studies on sub-bands 7, 8 and $9(6.33-7.92 \mathrm{GHz})$ of the MBOA solution (Fig. 1).

To improve the system performance, we propose a MC-SS scheme which consists in assigning to each user a specific block of subcarriers [5]. This scheme is applied to UWB while respecting the OFDM parameters of the MBOA solution. The spreading is applied in the frequency domain in order to improve the signal robustness against frequency selectivity of the UWB channel and against narrowband interference. The MC-SS system benefits from the performance and advantages of the MC-CDMA system while improving the resource allocation flexibility. With the MC-SS scheme, symbols are simultaneously transmitted by the same user on a specific subset of subcarriers and undergo the same distortions. Selfinterference (SI), which replaces the multiple-access interference (MAI) obtained with MC-CDMA, can be easily compensated by single user detection with only one complex coefficient per subcarrier.

A schematic representation of the sub-bands distribution of the MC-SS scheme is depicted in Fig. 2. At a given time, each user is allocated a group of 100 data subcarriers equivalent to one of the MBOA sub-bands of $528 \mathrm{MHz}$ bandwidth, in order not to increase the system complexity compared to MBOA. Each sub-band is then divided into several blocks $b$, each of them including a number of subcarriers equal to the spreading code length $L$. Note that in this figure, the subcarriers linked by the same spreading codes are adjacent to simplify the schematic representation, whereas in reality they are not necessarily.

The channel model used for our study is the one adopted by the IEEE 802.15.3a committee for the evaluation of UWB physical layer proposals [6]. It is a modified version of the

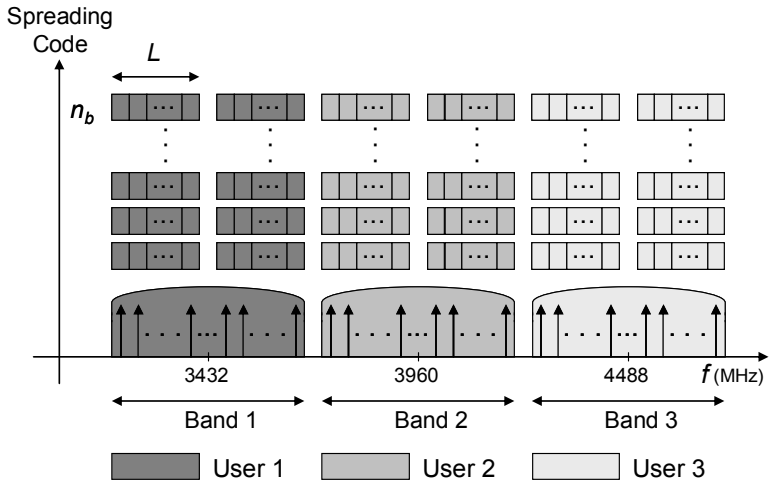

Fig. 2. Sub-bands distribution of the MC-SS scheme for 3 users.

Saleh-Valenzuela model for indoor channels, fitting the properties of measured UWB channels. A lognormal distribution is used for the multipath gain magnitude. In addition, independent fading is assumed for each cluster and each ray within the cluster. Moreover, four different channel models (CM1 to CM4) are defined for the UWB system modeling, each with arrival rates and decay factors chosen to match different usage scenarios and to fit line-of-sight (LOS) and non-line-of-sight (NLOS) cases.

\section{MC-SS SYSTEM OPTIMIZATION}

In this section, the objective is to properly handle the spreading process so that the system performance is improved. The spreading function brings some additional parameters which are the number $N$ and the length $L$ of the spreading sequences to use. These parameters constitute new degrees of freedom in terms of system configuration and can be optimally chosen as proposed in this part. In order to focus on the study of the precoding function, the only spreading and OFDM functions are considered here, and other functions of the global transmission chain are not taken into account.

The MC-SS system optimization is divided in 2 cases. First, we consider a fixed constellation order of QPSK as in MBOA and we optimize the number of codes in order to maximize the system range. Then, in order to get higher throughput, we propose a resource allocation algorithm with variable constellation orders.

The throughput in bits per symbol of an OFDM system is derived from Shannon theorem by

$$
R_{\text {OFDM }}=\sum_{k \in S} \log _{2}\left(1+\frac{1}{\Gamma}\left|h_{k}\right|^{2} \frac{E_{k}}{N_{0}}\right),
$$

where $S$ is the group of used subcarriers, $\Gamma$ the signal-tonoise ratio (SNR) gap, $h_{k}$ and $E_{k}$ the frequency-domain response and the transmitted power density of the $k^{\text {th }}$ subcarrier respectively, and $N_{0}$ the noise density.

The total throughput in bits per symbol of a MC-SS system using a zero-forcing (ZF) detection is given by [7] 


$$
R_{S S-M C-M A}=\sum_{b=1}^{B} \sum_{n=1}^{N_{b}} \log _{2}\left(1+\frac{1}{\Gamma} \frac{L^{2}}{\sum_{i=1}^{L} \frac{1}{\left|h_{i, b}\right|^{2}}} \frac{E_{n, b}}{N_{0}}\right),
$$

where $B$ is the number of blocks, $N_{b}$ the number of codes per block $b, L$ the spreading code length, $h_{i, b}$ the frequencydomain response of the $i^{\text {th }}$ subcarrier in block $b$, and $E_{n, b}$ the power density of code $n$ within block $b$, with the constraint

$$
\sum_{n=1}^{N_{b}} E_{n, b} \leq E \quad \text { and } \quad E_{n, b} \geq 0 \quad \forall b,
$$

where $E$ is the PSD limit defined by the FCC or the ECC.

\section{A. Fixed QPSK constellation}

We consider $K$ data subcarriers per sub-band and a fixed QPSK constellation as in the MBOA solution. For a fixed target throughput of $2 \mathrm{~K}$ bits per symbol, it is demonstrated in [8] that to maximize the noise margin of the MC-SS system, a code length $L$ equal to the total number of useful subcarriers $K$ should be used. Consequently, one single block system should be considered.

Now, we optimize the code length and the number of codes in order to maximize the system throughput and the system range when the throughput of $2 K$ bits per symbol is not reachable at high attenuation levels.

In a general approach with variable throughput, the number of codes can be lower than the code length. In this case, a multi-block configuration has to be considered and each block can exploit its own code length. But finding the optimal block sizes amounts to resolving a complex combinational optimization problem that can not be reduced to an equivalent convex problem. Then, no analytical solution exists and optimal solution can only be obtained following exhaustive search [7]. In order to avoid prohibitive computations, we assume a single block configuration system.

Maximizing the system range is equivalent to maximizing the system throughput. Using Lagrange multipliers to solve (2) under constraint (3), we find that the optimal solution which maximizes the non-integer system throughput would be to consider

$$
E_{n, b}=E / N_{b} \text { and } N_{b}=L, \forall b .
$$

Then, the optimal non-integer throughput for the single block system becomes

$$
R=L \log _{2}\left(1+\frac{L}{\sum_{i=1}^{L}\left(1 /\left|\hat{h}_{i}\right|^{2}\right)}\right),
$$

with $\left|\hat{h}_{i}\right|^{2}=\left|h_{i}\right|^{2} E / \Gamma N_{0}$ and $h_{i}$ the frequency-domain response of the $i^{\text {th }}$ subcarrier of the single block sub-band.
Theorem 1: With a QPSK constellation and a PSD constraint of $\sum_{n=1}^{L} E_{n} \leq E$, the optimal number of codes that can be used for a given spreading code length $L$ is $N=\left\lfloor L\left(2^{R / L}-1\right) / 3\right\rfloor$, with $R$ given by (4).

Proof: Let $N$ be the optimal number of spreading codes that maximizes the system range. Thus, when the system is using $N$ codes, the PSD constraint has to be respected, whereas when it is using $N+1$ codes, the constraint should not be respected anymore. Therefore, $N$ should satisfy

$$
\begin{aligned}
& \left\{\begin{array}{l}
E-\sum_{n=1}^{N} E_{n}=\frac{L}{\alpha}\left(2^{R / L}-1\right)-\frac{N}{\alpha}\left(2^{2}-1\right) \geq 0, \\
E-\sum_{n=1}^{N+1} E_{n}=\frac{L}{\alpha}\left(2^{R / L}-1\right)-\frac{N+1}{\alpha}\left(2^{2}-1\right)<0,
\end{array}\right. \\
& \alpha=L^{2} / \sum_{i=1}^{L} \frac{N_{0} \Gamma}{\left|h_{i}\right|^{2}} .
\end{aligned}
$$

with

From (5), $N \leq L\left(2^{R / L}-1\right) / 3$ and $N>L\left(2^{R / L}-1\right) / 3-1$. Hence, $N=\left\lfloor L\left(2^{R / L}-1\right) / 3\right\rfloor$.

From (4) and Theorem 1, since the number of codes can not be larger than the code length, the maximum reachable throughput for a given code length $L$ becomes

$$
\left.R(L)=2 \times \min \left\{\frac{L^{2}}{3 \sum_{i=1}^{L}\left(1 /\left|\hat{h}_{i}\right|^{2}\right)}\right\rfloor, L\right\} .
$$

Finally, the maximum reachable throughput with the optimal code length becomes

$$
R_{\max }=\max _{1 \leq L \leq K}\{R(L)\} .
$$

A low-complexity algorithm that derives the optimal number of codes $N$ is applied to the MC-SS system using a fixed QPSK constellation. This algorithm can be advantageously exploited for high attenuation levels since it increases the system range significantly when the channel response is critical, as shown in Section IV. In addition, these improvements can be obtained without changing the radiofrequency front-end of the MBOA solution.

\section{B. Variable constellations}

Here, we move away from the MBOA proposal by considering variable constellations (QPSK, 8-QAM and 16-QAM) in order to reach higher throughput. We propose a resource allocation algorithm that distributes energy, bits and codes efficiently across the spectrum in order to maximize the total system throughput under a PSD constraint.

By considering a multi-block context, the maximum reachable non-integer throughput in bits per block $b$ is derived from (4) 


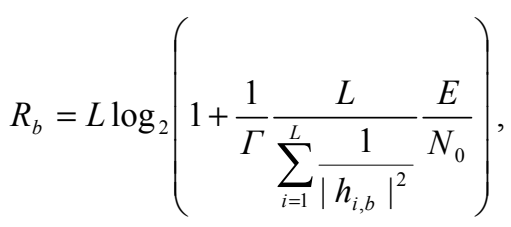

and the maximum throughput in bits per code $n$ becomes

$$
R_{n, b}=R_{b} / L \quad \forall n \in[1: L] .
$$

Since we have discrete constellation orders, the throughput per code must always have integer values. An optimal solution for this problem is given in [7]. It shows that an optimal throughput distribution would be to allocate $\left\lfloor R_{b} / L\right\rfloor+1$ bits to $m$ codes and $\left\lfloor R_{b} / L\right\rfloor$ bits to the remaining $L-m$ codes, where $m$ is an integer number given by

$$
m=\left\lfloor L\left(2^{R_{b} / L\left\lfloor\left\lfloor R_{b} / L\right\rfloor\right.}-1\right)\right\rfloor .
$$

Hence, the discrete throughput in bits per code becomes

$$
\begin{cases}\bar{R}_{n, b}=\left\lfloor R_{b} / L\right\rfloor+1 & \forall n \in[1: m] \\ \bar{R}_{n, b}=\left\lfloor R_{b} / L\right\rfloor & \forall n \in[m+1: L] .\end{cases}
$$

The power density per code for block $b$ is then given by

$$
\bar{P}_{n, b}=\frac{\Gamma}{L^{2}} \sum_{i=1}^{L} \frac{N_{0}}{\left|h_{i, b}\right|^{2}}\left(2^{\bar{R}_{n, b}}-1\right),
$$

and the total discrete throughput in bits per block $b$ becomes

$$
\bar{R}_{b}=\left\lfloor L\left(2^{R_{b} / L-\left\lfloor R_{b} / L\right\rfloor}-1\right)\right\rfloor+L\left\lfloor R_{b} / L\right\rfloor .
$$

This algorithm increases the system throughput considerably when applied to an MC-SS scheme with variable constellations. Consequently, the MC-SS system range is improved compared to MBOA and the adaptive system is able to transmit data at very low attenuation levels.

\section{SIMULATION RESULTS}

In this section, we present the simulations performed on sub-bands 1, 2 and 3 (American context, 3.16-4.75 GHz) and sub-bands 7, 8 and 9 (European context, 6.33-7.92 GHz) of the MBOA solution.

Fig. 3 and Fig. 4 represent the results of the throughput optimization for a fixed QPSK constellation in the American context. The total throughput per OFDM symbol of a single user over CM1 channel model is given in Fig. 3, for different channel attenuation levels. With the MBOA solution, the total throughput of $200 \mathrm{bit} / \mathrm{symbol}$ is not reachable at attenuation levels higher than $46 \mathrm{~dB}$, whereas with the proposed MC-SS scheme using a single block of length $L=100$, we are able totransmit $200 \mathrm{bit} / \mathrm{symbol}$ until a $61 \mathrm{~dB}$ level $(15 \mathrm{~dB}$ larger range). Moreover, when we optimize $L$ and $N$ for the MC-SS system, we are able to transmit data at much higher attenuations (until $84 \mathrm{~dB}$ ), and the reachable range with adaptive MC-SS is always larger than the range of an adaptive OFDM system. With the called adaptive OFDM scheme, the number of QPSK modulated subcarriers can vary from 100 to 0 , whereas with the MBOA solution the number of active subcarriers is always equal to 100 . The optimal values of $L$ and $N$ that maximize the range of the adaptive MC-SS system are given in Fig. 4. We can notice that at high attenuations, the optimal value of $N$ is not necessarily equal to $L$. Hence, the algorithm is very efficient at high attenuation levels. Moreover, the radio-frequency front-end of the MBOA solution is maintained.

Fig. 5 and Fig. 6 represent the throughput of a single user when variable constellation orders are considered (QPSK, 8-QAM and 16-QAM), for the American and European contexts, respectively. When the resource allocation algorithm is applied to the MC-SS scheme, the throughput is equal to $400 \mathrm{bit} / \mathrm{symbol}$ at low attenuation levels. At high attenuation levels, the adaptive MC-SS range is always larger than the adaptive OFDM range (around $10 \mathrm{~dB}$ at critical attenuation levels). This is due to the energy gathering capability of MC-SS which can exploit, contrarily to OFDM, the residual energy conveyed by each subcarrier. Similar results are obtained for the different channel models (CM1 to CM4). In this case, since we are using 8-QAM and 16-QAM constellations, one or two additional bits are needed for the analog-to-digital conversion. Hence, the radio-frequency front-end complexity is not increased significantly compared to MBOA.

\section{CONCLUSION}

In this paper, we proposed a multicarrier spread-spectrum waveform which is new for high data rate UWB applications and which respects the OFDM parameters of the MBOA solution in order not to increase the system complexity significantly. First, while considering a fixed QPSK constellation, the spreading code length and the number of codes were optimized to maximize the system range. Furthermore, we used variable constellation orders and we proposed a low-complexity resource allocation algorithm in order to maximize the system throughput. We showed that the adaptive MC-SS system is able to transmit information at attenuation levels much higher than the attenuation limits of the MBOA solution. These optimizations did not take into account the channel coding scheme. However, the performance comparison of the final systems with channel coding shows that our proposed adaptive MC-SS scheme can be advantageously exploited for high data rate UWB applications. In addition, the proposed system complexity is not increased significantly compared to MBOA. 


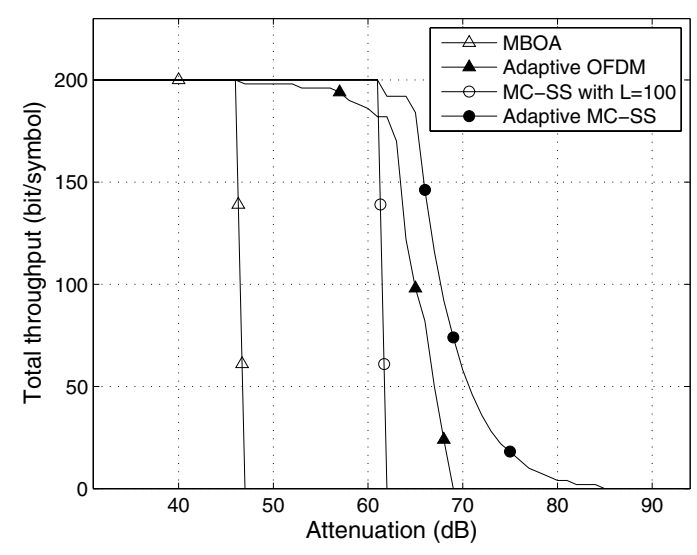

Fig. 3. System throughput with the optimal number of codes and a fixed QPSK constellation, over CM1 model (American context).

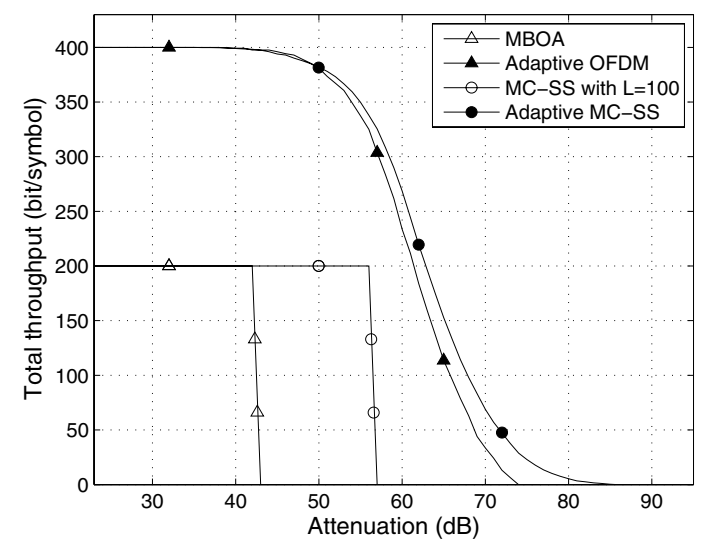

Fig. 5. System throughput with variable constellation orders, after applying the resource allocation algorithm over CM2 model (American context).

\section{ACKNOWLEDGEMENT}

The authors would like to thank France Télécom R\&D/RESA/BWA which supports this study within the contract 46136582.

\section{REFERENCES}

[1] "First report and order, revision of part 15 of the commission's rules regarding ultra-wideband transmission systems," FCC, ET Docket 98-153, Feb. 14, 2002.

[2] IEEE P802.15 Working Group for Wireless Personal Area Networks (WPANs), "Multi-band OFDM physical layer proposal for IEEE 802.15 task group 3a," Sept. 2004.

[3] M. Schmidt and F. Jondral, "Ultra wideband transmission based on MC-CDMA," in Proc. IEEE Global Commun. Conference, Vol. 2, pp. 749-753, Dec. 2003.

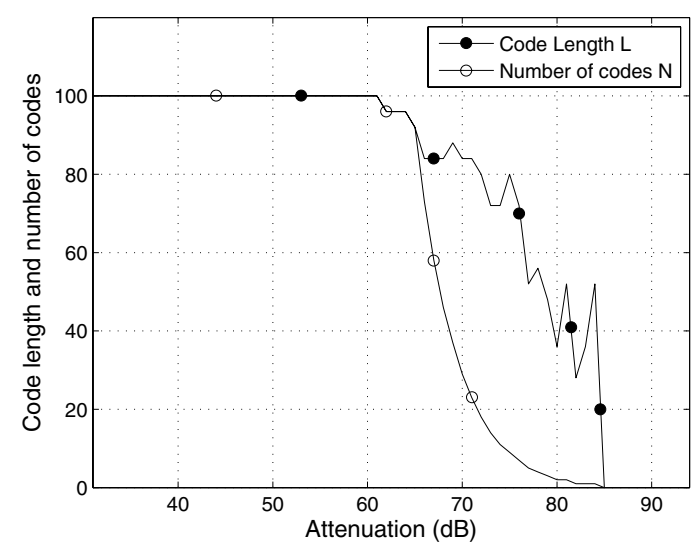

Fig. 4. Optimal adaptive MC-SS configuration with a fixed QPSK constellation, over CM1 model (American context).

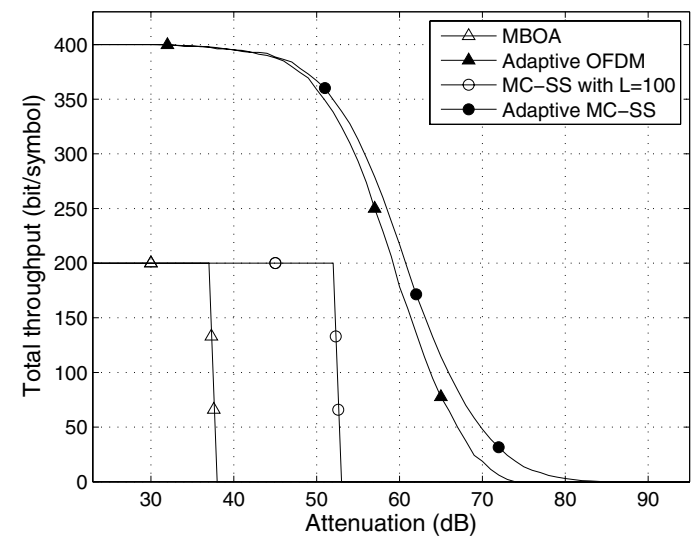

Fig. 6. System throughput with variable constellation orders, after applying the resource allocation algorithm over CM3 model (European context).

[4] "ECC Decision of 24 March 2006 on the harmonised conditions for devices using Ultra-Wideband (UWB) technology in bands below 10.6 GHz," ECC/DEC(06)04, March 2006.

[5] S. Kaiser and K. Fazel, "A flexible spread-spectrum multicarrier multiple-access system for multi-media applications," in Proc. IEEE Intern. Symposium on Personal, Indoor and Mobile Radio Commun. (PIMRC'97), Finland, Sept. 1997.

[6] J. Foerster et al., "Channel modeling sub-committee report final," IEEE802.15-02/490, Nov. 2003.

[7] M. Crussière, J-Y. Baudais and J-F. Hélard, "Adaptive linear precoded DMT as an efficient resource allocation scheme for power line communications," in Proc. IEEE Global Commun. Conference, San Francisco, USA, Dec. 2006.

[8] A. Stephan, J-Y. Baudais and J-F. Hélard, "Adaptive multicarrier spread-spectrum with dynamic time-frequency codes for UWB applications," in Proc. IEEE Workshop on Multi-Carrier Spread Spectrum (MC-SS'07), pp. 197-206, Germany, May 2007. 